\title{
Cultural Issues Related to ICD-11 Mental, Behavioural and Neurodevelopmental Disorders
}

\author{
Культуральные аспекты психических и поведенческих \\ расстройств и нарушений нейропсихического развития в МКБ-11 \\ doi:10.17816/CP67
}

\section{(C) Pratap Sharan, Gagan Hans}

The Department of Psychiatry All India Institute of Medical Sciences, New Delhi, India

\section{(C) Пратап Шаран, Гаган Ханс}

Департамент психиатрии, Всеиндийский институт медицинских наук, Нью Дели, Индия

\section{ABSTRACT}

The challenge of producing a classificatory system that is truly representative of different regions and cultural variations is difficult. This can be conceptualized as an ongoing process, achievable by constant commitment in this regard from various stakeholders over successive generations of the classificatory systems. The objective of this article is to conduct a qualitative review of the process and outcome of the efforts that resulted in the ICD-11 classification of mental, behavioural and neurodevelopmental disorders becoming a global classification. The ICD-11 represents an important, albeit iterative, advance in the classification of mental, behavioural and neurodevelopmental disorders. Significant changes have been incorporated in this regard, such as the introduction of new, culturally-relevant categories, modifications of the diagnostic guidelines, based on culturally informed data and the incorporation of culture-related features for specific disorders. Notwithstanding, there are still certain significant shortcomings and areas for further improvement and research. Some of the key limitations of ICD-11 relate to the paucity of research on the role of culture in the pathogenesis of illnesses. To ensure a classificatory system that is fair, reliable and culturally useful, there is a need to generate empirical evidence on diversity in the form of illnesses, as well as mechanisms that explain these in all the regions of the world. In this review, we try to delineate the various cultural challenges and their influences in the formulation of ICD-11, along with potential shortcomings and areas in need of more improvement and research in this regard.

\section{АННОТАЦИЯ}

Задача создания классификационной системы, которая действительно бы учитывала региональные и культуральные различия, является достаточно сложной. Это долгий процесс, ориентированный на последующие изменения классификации, с участием всех заинтересованных сторон. Цель данной статьи - представить содержательный обзор этапов разработки и результатов усилий, которые привели к тому, что классификация психических, поведенческих расстройств и нейропсихического развития в МКБ-11 становится поистине глобальной, т.е. применимой во всем мире. МКБ-11 делает важный очередной шаг вперед в развитии классификации. Это связано с такими значимыми изменениями, как внесение новых культурально значимых категорий, обновление диагностических указаний в соответствии с данными, полученными в разных странах, и учет культуральной специфики определенных расстройств.

Несмотря на это, все еще имеются некоторые существенные недостатки и вместе с тем возможности для развития и проведения исследований. Некоторые из ключевых ограничений МКБ-11 связаны 
с недостаточным изучением роли культуры в патогенезе заболеваний. Для обеспечения ясной, надежной и полезной с учетом культурного контекста классификационной системы необходимо собрать воедино эмпирические доказательные данные о разнообразии проявлений болезней, а также их патогенеза, в разных регионах мира. В этом обзоре делается попытка обозначить изменения МКБ-11, связанные с различными культуральными аспектами, а также потенциальные недостатки и пути дальнейшего совершенствования с опорой на исследовании в этом направлении.

Keywords: ICD-11; WHO; culture; mental disorders; classification

Ключевые слова: МКБ-11; ВОЗ; культура; психические расстройства; классификация

\section{INTRODUCTION}

The establishment, maintenance and revision of the International Classification of Diseases and related health problems (ICD) is a core constitutional responsibility of the World Health Organization (WHO). Revisions are undertaken at regular intervals to keep abreast of the recent scientific advances in understanding various disorders.

Cultural considerations are important in terms of classification, as they improve diagnostic assessments when cultural issues are involved, reduce iatrogenic harm resulting from the misdiagnosis of cultural problems, improve the treatment of such problems by stimulating clinical research and encourage clinical training centres to address the cultural dimensions of human existence. However, an international classification system has to strike a pragmatic balance between the need for a universal classificatory system that can facilitate the reliable communication of clinical information across geographic and cultural boundaries, while retaining the ability to be contextually and culturally relevant during the clinical encounter, ${ }^{1}$ as this encounter translates into health information and health action.

A universalizing approach is detrimental to health information since the multiplicity of cultural expressions of mental disorders lead to difficulties in diagnoses, as exemplified by a 34-fold difference in the prevalence of social anxiety disorder in various cross-national studies, utilizing similar methodologies ${ }^{2}$ and by markedly different prevalence rates for ADHD in the regions, utilizing the ICD and DSM classification system. ${ }^{3}$ Although these differences could be due to multiple reasons, it is possible that the guidelines that are based on the experiences of a few cultures, fail to capture the expression of the disorders in others. In addition to difficulties in correctly labelling/diagnosing cultural variants of mental disorders, treating sociocultural manifestations and processes as epiphenomenal, may impact on the understanding of the etiological role of cultural factors in the development of mental disorders. ${ }^{4}$ Ignoring the sociocultural aspects of mental illness may have scientific consequences, however, equally important are the social justice repercussions of this approach, e.g., the risk of misdiagnosis and perpetuation of clinical stereotypes, based on race, ethnicity, gender, religion and sexual orientation. ${ }^{5}$ There is, therefore, a need to explore the alternate symptom expressions, variations and overlap between the different disorders, risk moderation and exploratory models in diverse cultures for a comprehensive and inclusive nosology. ${ }^{6}$

The objective of this article is to conduct a qualitative review of the process and outcome of the efforts that resulted in the ICD-11 classification of mental, behavioural and neurodevelopmental disorders becoming a global classification.

\section{ATTENTION TO CULTURAL ISSUES IN ICD-11}

The ICD-10 Clinical Descriptions and Diagnostic Guidelines (CDDG) has previously noted the presence of cultural variations in the expression of disorders under broad disorder groupings (e.g., somatoform disorder) and in help-seeking and illness-related behaviours. However, considerations related to culture were not systematically incorporated in the manual. ${ }^{1,7}$ This led to a situation in which a number of national and regional adaptations were proposed to address cultural variations in the expression of mental disorders, including the Chinese Classification of Mental Disorders, the Japanese Clinical Modification of the ICD-10, the Latin American Guide for Psychiatric Disorders and the Cuban Glossary of Psychiatry. ${ }^{8}$ 
The ICD-11 has been developed for global application. Reflecting the cultural context in which clinical encounters take place is likely to enhance this goal. Accordingly, ICD-11 has emphasized cultural considerations as impacting all patient encounters rather than focusing on a few exotic (and rare) culture bound syndromes.

\section{THE PROCESS OF ICD-11 DEVELOPMENT}

Cultural considerations in the overall developmental process of the ICD-11

The WHO implemented several strategies to enhance the cultural applicability of the ICD-11 mental and behavioural disorders classification. The first strategy was an international and multilingual review of the literature to evaluate major trends, themes and areas of active debate related to the classification of mental disorders, in particular, with regard to clinical utility in low- and middle-income countries ${ }^{9}$ (LMIC). ${ }^{*}$ This was carried out in addition to gathering information and recommendations on the alternate descriptions of various disorders. A systematic analysis of country-level and regional diagnostic systems for mental disorders was also conducted along these lines. For example, the Third Cuban Glossary of Psychiatry included several categories, not featured in the ICD-10, which focus specifically on problems related to functioning in the family environment (e.g., among people with intellectual disabilities). ${ }^{10}$ This is consistent with a strong cultural emphasis on family in Cuba, as compared with the USA or Western Europe but may also have significant utility for treatment planning, given that the family is likely to be a key vehicle for support and social inclusion, in many countries. The second strategy was significant engagement of the diverse constituency groups and consultation with the representatives of various geographical regions of the world. ${ }^{11}$ This information was supplemented with surveys of psychiatrists ${ }^{12}$ and psychologists ${ }^{13}$ in collaboration with other leading organizations, including the World Psychiatric Association (WPA). The third strategy was to adopt greater flexibility in the description of the various psychiatric disorders to make them more inclusive for varying presentations. ${ }^{14,15}$ This strategy also advocated the utilization of prototypical descriptions, as opposed to lists of criteria, to facilitate the inclusion of cultural variations, as well as contextual and health system factors, affecting diagnostic practice. ${ }^{16}$ The fourth strategy was to conduct a series of systematic field studies, focusing on clinical utility and global applicability through a network of field study centres in large LMICs.9, ${ }^{915}$ In addition, the field testing of ICD-11 has further enhanced the validity and reliability of various disorders, cutting across cultural groups globally, with regard to its accuracy, consistency and clinical utility. All these developments led to the infusion of sociocultural perspectives, with structural features to support the cultural utility of ICD-11..$^{14,17}$

\section{ICD-11 Working group focused on cultural influences}

The WHO constituted a working group, with the primary aim of developing cultural guidance for the ICD-11 CDDG. The 'ICD-11 Working Group on Cultural Influences' formulated certain relevant questions for eliciting the factors which account for cultural variations that were related to: (1) the influence of culture on the presentation of disorders and the mechanisms thereof, (2) whether the differences in the prevalence of various disorders between populations could be attributed to cultural factors and linked to cultural mechanisms and (3) the identification of cultural concepts of distress (idioms, syndromes, explanations/causes) in various cultural groups, which are related to various disorders. ${ }^{1}$ An example of the recommendations that emerged from this exercise is presented in Box 1.

\section{Discussions in workgroups on specific disorders} The fact that classification in psychiatry is still essentially based on the best judgement of a group of experts, who tend to rely on data, largely generated from the western populations, make its global applicability questionable. ${ }^{18}$ The WHO has sought to include a significant number of members in the different working groups from LMIC, to tap into diverse cultural experiences for inclusive decision-making. 11,19,20 An example of recommendations that emerged from discussions within various workgroups is highlighted in Box 2.

* WHO Member States are grouped into four income groups [low, lower-middle, upper-middle and high] based on the World Bank list of classification of economies, which is based on the gross national income per capita estimates ${ }^{9}$ 


\section{Box 1: cultural considerations for adjustment disorder:}

- Adjustment disorder may be exacerbated by limited family or community support, particularly in collectivistic or sociocentric cultures. In these societies, the focus of the worry may extend to stressors, affecting close relatives or friends.

- $\quad$ Adjustment disorder reactions that include dissociative symptoms may be more prominent in certain cultural groups.

- $\quad$ Symptoms of the disorder may be influenced by local idioms (e.g., susto or espanto [fright] in Central America) that are associated with fear or subsequent worry regarding a stressor with strong cultural connotations (e.g., becoming suddenly frightened when crossing an unpopulated area alone at night). These idioms are also applicable to anxiety disorders.

\section{Box 2: cultural variant of rumination-regurgitation disorder: deliberationof the workgroup on eating disorders}

A case vignette from South Asia was presented to the work group that did not fit the modal presentation of rumination disorder, along with relevant literature from the region..$^{21-23}$

The case: MS, is a 16-year-old boy. He was referred form the gastroenterology outpatient department because of 'persistent vomiting' and loss of weight over a two-year period for which medical-surgical causes could not be established.

He presented with a history of 'vomiting' after meals, initially after an occasional meal and, subsequently, after each meal. He reported a sense of fullness and pain in his abdomen after meals but no nausea. The 'vomiting' was spontaneous and was preceded either by no or minimal retching and was described as 'a filling up of the mouth by the recently consumed food/beverages.' He never induced 'vomiting' and did not try to vomit in secret. The contraction of the abdominal muscles to facilitate 'vomiting' was reported in the initial six months, but 'vomiting' became automatic with the passage of time. He had cut down on food "to avoid vomiting", to $25 \%$ of his usual intake. His weight at the time of admission was 40 kilograms and he had lost $37 \%$ of his weight, compared with his premorbid state. His BMI was 14.28 . He had stopped interacting with anyone other than his immediate family and had not attended school for the last 18 months, because of persistent 'vomiting.'

The patient did not consider himself overweight at his premorbid weight (62 kilograms). He used to exercise regularly before the onset of illness and had continued to do so for the initial six months, when he was 'vomiting' occasionally after meals. Though, he did not report any dismay at his current emaciation, he accepted that an effort to increase his weight was justified. He denied binge eating or a conscious motivation to diet, use of laxatives or diuretics. He did not report sustained sadness or depressive thoughts. He had complained of aches and pains for the last 18 months and had almost stopped walking without assistance for the last three months.

Discussion in the working group: MS would not meet the requirement for a typical case of rumination disorder in ICD-11, because although the food comes back up without retching, it is not re-chewed and re-swallowed, or spat out. It is not held in the mouth for any length of time and does not go up and down the oesophagus. Instead, as in the case of vomiting, it comes up and is expelled in one movement.

The solution: In the culture-related features (as known and relevant) for rumination disorder, it was clarified that: certain cases of what has been considered to be 'psychogenic vomiting', particularly in South Asia, may actually be cultural variants of rumination disorder, and the latter should be regarded as a differential diagnosis, in cases of psychogenic vomiting.

This cultural variant of rumination disorder is characterized by repeated regurgitation of food, that is usually associated with the emptying of the mouth, rather than re-chewing or re-swallowing. Initially, individuals with this disorder seem to volitionally (usually by contracting abdominal muscles) (as inferred from detailed clinical evaluation of their behaviour) and repeatedly bring up partially digested food back into the mouth (i.e., regurgitation) after being previously swallowed, with relative ease; there is minimal physical discomfort or anxiety, associated with this behaviour. Rumination disorder should only be diagnosed if the behaviour is frequent (at least several times per week), occurs over a period of several weeks (e.g., at least four weeks). The diagnosis of rumination disorder should not be made in the context of an associated medical condition (e.g., oesophageal strictures or neuromuscular disorders affecting oesophageal function), when the medical condition wholly accounts for the behavioural symptoms. Subsequently, effortless regurgitation seems to become automatic, and at times, progresses to regurgitating the entire meal after most meals. If substantial weight loss occurs, evaluation for anorexia nervosa may need to be considered. Compared to psychogenic vomiting, rumination disorder is diagnosed when the regurgitation is relatively effortless in nature and appears to be volitional, at least in the early stages of the disorder. 


\section{Compatibility}

of ICD-11 with DSM=5

Both ICD-11 and DSM-5 accept that culture plays an important role in the distress, perceptions, coping, support and help-seeking for all patients.

Efforts were also undertaken to make ICD-11 and DSM-5 more compatible with one other in terms of the definitions of mental disorders. ${ }^{24,25}$ For example, both ICD-11 and DSM-5 exclude the culturally approved responses to common stressors or losses, such as bereavement and social deviation. ${ }^{11}$ These have been mentioned in the definition of the mental disorders in DSM-5; in ICD-11, they have been highlighted in the diagnostic guidelines for the specific disorders, namely, distinguishing bereavement reactions from depression and socially stigmatized sexual behaviours from paraphilic disorders.

\section{THE PRODUCT}

Introduction of culturally relevant new categories The approach, adopted in ICD-11, in which greater significance has been given to the data from the LMICs, has resulted in the inclusion of certain newer categories of disorders. The understanding is that these can result in better recognition of the transcultural representation of such a group of disorders. An example is given in Box 3.

\section{Modification of diagnostic guidelines,}

\section{based on culturally informed data}

Certain modifications have been made to the diagnostic guidelines, to ensure wider applicability in the different regions of the world, as in the case of social anxiety disorder (Box 4).

\section{Culture-related features for specific disorders}

There is a dedicated section in the accompanying text relating to the cultural considerations for all the disorders in ICD-11, which summarizes information on cultural variations in terms of describing distress, symptom patterns, dysfunctions and course, with a view to promoting a culturally sensitive application. ${ }^{16}$ Certain examples are given in box 5 . The focus, here, was on providing pragmatic, actionable material to assist clinicians in their evaluation of patients, using the ICD-11 guidelines and reducing bias in clinical decision-making, by facilitating diagnostic assessment in a culturally informed manner. ${ }^{1}$

\section{POTENTIAL SHORTCOMINGS IN NEED OF IMPROVEMENT OR AREAS IN NEED FOR RESEARCH}

Omitted disorders

Certain ICD-10 disorders that were commonly diagnosed in LMICs have been omitted from ICD-11. This could lead to diagnostic, treatment and research uncertainty, as well as causing coding difficulties. Some examples are mentioned in Box 6.

\section{Box 3: examples of culturally relevant new categories}

Avoidant/restrictive food intake disorder (ARFID)

ARFID and anorexia nervosa are both characterized by dietary restriction or food avoidance but the core disturbance in terms of experiencing the body weight or shape, is absent in patients with ARFID. ${ }^{26}$ The factors contributing to the eating disturbance in ARFID, include little interest in eating and/or avoidance of multiple food types. The avoidance of specific food types may be based on sensory properties or on perceived adverse consequences. The importance of somatic factors has emerged, as a result of descriptions provided by LMICs. ${ }^{23}$

Box 4: example of modification of diagnostic guidelines, based on culturally informed data

Social anxiety disorder

It has been established in cross cultural research with taijin kyofusho in Japan and Taein kong po in Korea, that as part of the symptomology of the social anxiety disorder, the fear of negative evaluation by others can take the form of fear that the individual may offend others in addition to or instead of fear that the person will feel embarrassed or humiliated, as a result of engaging in the social behaviour. Similar findings of the fear of offending others in social anxiety disorder, has also been found in certain studies in western settings. ${ }^{28}$ The modifications in the diagnostic guidelines for social anxiety disorder allow for inclusion of these varied transcultural presentations of social anxiety in ICD-11.11 


\section{Box 5: examples of culture-related features for specific disorders}

\section{Depression}

Although the symptoms of sadness and anhedonia have been retained as the principal symptoms of depression in ICD-11, clinicians are informed in this section that the somatic symptoms can predominate among patients with depression. This has been demonstrated in studies from LMICs and there may be significant cultural variability as to whether and how patients discuss their emotions with their clinicians.

Post-traumatic stress disorder

Culture-related features in PTSD in ICD-11 state that culturally sanctioned and recognized expressions or idioms of distress, explanatory beliefs and cultural syndromes, may be a key element of the trauma response. These may influence the symptomatology and comorbidity, particularly through somatization, as well as having emotional, cognitive and behavioural expressions of distress. These cultural-related features have been based on a number of observations, particularly among patients from LMICs. For example, cultural idioms of distress commonly present as somatic symptoms, such as ohkumlang (tiredness) and bodily pain among tortured Bhutanese refugees or as possession states in countries like Guinea Bissau, Mozambique and Uganda. This may take the form of susto (fright) among Latino populations, as kit chraen (thinking too much) and sramay (flashbacks of past traumas in the form of dreams and imagery that pervade one's waking life) in Cambodia. All these cultural idioms can influence the presentation and interpretation of PTSD among the affected populations. ${ }^{16}$

\section{Box 6: examples of ICD-10 disorders omitted in ICD-11}

\section{Neurasthenia}

The ICD-11 has proposed a simplified category relating to bodily distress disorders to replace all the categories within the group of somatoform disorders, with the exception of hypochondriasis. This simplification of the diagnostic category may result in over-inclusiveness of various cultural phenomena, within the ambit of this category.

Though virtually unused in western countries, neurasthenia was for many years by far the most commonly diagnosed mental disorder in outpatient and community settings in China. ${ }^{29}$ The Chinese conceptualization of neurasthenia attaches equal diagnostic weight to somatic, cognitive and emotional symptomatology, and in this respect differs from western diagnostic constructs. A wider application of western classification systems in Chinese psychiatric research, has contributed to the marginalization of neurasthenia as a residual somatoform category in the specialist mental health sector. The fact that the diagnostic category of neurasthenia is still widely used by general physicians and psychiatric practitioners, and is also widely understood by lay people in both urban and rural China, ${ }^{29}$ suggests that it has continuing clinical utility that should be examined further.

Psychogenic vomiting

Psychogenic vomiting has been removed from ICD-11 as a diagnostic entity, as it is not clear whether it is a mental disorder. While, certain cases of psychogenic vomiting would be diagnosed as cultural variants of rumination disorder (Box 2), other cases of psychogenic vomiting would now be diagnosed as an unspecified eating disorder or as cyclical vomiting (not a mental disorder).

Psychogenic vomiting is the most common eating disorder diagnosis among psychiatric service users in the Indian subcontinent. However, eating disorders like anorexia nervosa, bulimia nervosa and binge eating disorders are uncommon in clinical, as well as community samples. ${ }^{30}$ In a chart review of cases of eating disorders in a tertiary care centre in South India, $85.4 \%$ were diagnosed as having psychogenic vomiting and $14.6 \%$ as having anorexia nervosa. ${ }^{31}$ The female to male ratio for psychogenic vomiting (1.33\%) was less than that for anorexia nervosa (5\%).

Psychogenic vomiting can be a highly disabling condition ${ }^{32}$ that is often misdiagnosed. ${ }^{33}$ Under-recognition of this disorder can lead to delayed treatment, as well as affecting research efforts. The brain-gut may be involved in the modulation of stress, resulting in unexplained nausea and vomiting, and the association between these needs to be investigated. ${ }^{34,35}$

\section{Lack of focus on implementation and the client} Significant changes have been made in ICD-11 for inclusion of cultural variables in the nosology. However, guidance on implementation, training and application in diverse settings remain to be fully addressed.

While social science research has demonstrated the importance of culture in shaping psychiatric illness, clinical methods for assessing the cultural dimensions of illness, have not been adopted as part of routine care. The reasons for limited integration include the impression that attention to culture requires specialized skills, is only relevant to a subset of patients from unfamiliar backgrounds and is too time consuming to be useful. In the DSM-5, the Outline for Cultural Formulation (OCF) provides a framework for clinicians to organize cultural information, relevant to diagnostic assessment and 
treatment planning. The Cultural Formulation Interview (CFI) operationalizes the process of data collection for the OCF. A key goal of the CFI is to place the experience of the patient at the centre of the encounter, allowing the clinician to appreciate the personal, interpersonal and larger social contexts in which the problem, its interpretation and clinical presentation, emerge. A framework for the collection of cultural and individualized information, may facilitate culturally competent encounters. However, the ICD-11, as a classification for all illnesses is not intended to provide support for individual evaluation, including for psychiatric purposes. Clinicians wishing to assess cultural issues for ICD-11 could use interviews like the DSM-5 $\mathrm{CFI}$, Brief Cultural Interview, ${ }^{36}$ the McGill Illness Narrative Interview ${ }^{37}$ or other approaches, ${ }^{38}$ along with the ICD-11.

Furthermore, the cultural context and/or clinician values may impact diagnosis regarding cultural issues. Clinicians' awareness of and training relating to the diagnostic implications of cultural issues are necessary, as they may impact potential prognosis. Encouraging clinicians' selfawareness, in addition to being knowledgeable in relation to diversity factors, can aid in furthering diagnostic accuracy. However, this may require the incorporation of the concept of culture in the general training of mental health and primary care professionals.

\section{Lack of guidance on normal cultural variations}

Cultural issues may become pertinent for classification and diagnosis in multiple ways. The ICD-11 provides guidance on the assessment of pathological cultural symbols and expressions (e.g., religious delusions, trance and possession). However, clinicians may also have to manage cases with non-pathological cultural issues, which would be coded under 'Factors influencing health status and contact with health services ( $Z$ codes in ICD-10)' (e.g., life-cycle transitions, acculturation difficulties, issues related to sexual attitude, behaviour and orientation, mystical experiences, etc.). Cases may involve concurrent mental disorder with non-pathological cultural issues (e.g., obsessive-compulsive disorder with religious rituals), whereby $Z$ codes may be used in addition to mental disorder diagnosis. ICD-11 describes such conditions but does not provide guidance on their differentiation from pathology and labelling.

The incorporation of cultural consideration in classification should shift from an exclusive focus on pathology (differential diagnosis, source of pathology) to an understanding of the client's current issues and methods for treating them appropriately. ICD-11 has not adequately addressed this shift. However, an issue in this regard is the limited research available on markers of or criteria for pathology in cultural phenomena (e.g., intense focus on sin vs. scruples). In addition, there is a need to understand how practitioners are utilizing the $Z$ Codes for assessment of cultural issues.

\section{Alternate conceptualizations}

One of the limitations of the international nosology classificatory systems, is the fixation of phenomenological boundaries of the disorders, leading to the exclusion of culturally/contextually influenced variants of symptom expression. ${ }^{28}$ As the cartesian mind-body distinction is not recognized worldwide, as suggested by the conceptualization of neurasthenia in China, alternate models regarding the separation of affective disorders, anxiety and somatoform, could be evaluated for validity. ${ }^{39}$ Similarly, cultural concepts which overlap with multiple diagnoses like ataques de nervios (with panic disorder, posttraumatic stress disorder, intermittent explosive disorder) ${ }^{28}$ and dhat syndrome (health anxiety, somatoform, depressive and anxiety disorders $)^{40}$ could be evaluated as alternate formulations, that may be more valid for capturing the relevant phenomena (in terms of expression, as well as mechanism) within specific cultures.

At the same time, we need to recognize that the global mental health push, including the classificatory systems, are also influencing the cultural diversity in approaches to health and illness. The previously western syndrome of "depression" is becoming a master narrative among clinicians in diverse communities, where cultural syndromes are disappearing (e.g., neurasthenia in China, dhat syndrome in India, Hwabyung in Korea, and Taijin-kyofusho in Japan). The hybridization of cultures may alter the shape of alternate formulations.

\section{CONCLUSIONS}

A truly culturally sensitive classification of mental disorders is difficult to achieve for global use. The ICD-11 represents an important, albeit iterative, advance in this regard. The various changes made in the ICD-11 have added a consistent cultural lens to the diagnostic classification. The guidance for cultural considerations in ICD-11 should enhance the clinical utility of the constituent diagnostic 
constructs and help clinicians make culturally informed decisions. However, the limitations of ICD-11 with regard to cultural praxis also have to be understood.

Certain limitations of ICD-11 are related to the paucity of research on the role of culture in the pathogenesis of illnesses in non-western cultures. For a classificatory system that is fair, reliable and culturally useful, there is a need to generate empirical evidence on diversity, as well as mechanisms that explain these from the perspectives of all the regions around the world. ${ }^{41}$ This requires a strengthening of the research base for culture informed studies in LMICs, so they can better participate in the development of a culturally-fair, global classificatory system. Future research on the cultural framework of psychiatric conditions is not only important in better understanding these conditions but also makes the classificatory systems more acceptable globally.

Finally, there is a need to understand the limits of a cultural approach to health, which does not systematically address the range of social structural determinants (e.g., political and economic contexts) of health, but may be equally as important for clinical assessment and intervention in terms of cultural knowledge. ${ }^{42}$

Authors contribution: Pratap Sharan: Literature review, Article writing; Gagan Hans: Literature review, Article writing.

Conflict of interests: Authors declare no conflict of interest.

Funding: No source of external funding.

Informed consent of patients: Not Applicable.

\author{
Compliance with principles of bioethics: \\ Not Applicable.
}

\section{Correspondence to:}

\section{Professor Pratap Sharan, MD, PhD}

pratapsharan@gmail.com

\section{For citation:}

Sharan P, Hans G. Cultural issues related to ICD-11 mental, behavioural and neurodevelopmental disorders. Consortium Psychiatricum. 2021;2(2):7-15. doi:10.17816/CP67

\section{References}

1. Gureje O, Lewis-Fernandez R, Hall BJ, Reed GM. Systematic inclusion of culture-related information in ICD-11. World Psychiatry. 2019;18(3):357-358. doi:10.1002/wps.20676

2. Lewis-Fernandez R, Hinton DE, Laria AJ, et al. Culture and the anxiety disorders: recommendations for DSM-V. Depress Anxiety. 2010;27(2):212-229. doi:10.1002/da.20647

3. Achenbach TM, Rescorla LA. Multicultural Understanding of Child and Adolescent Psychopathology: Implications for Mental Health Assessment. Guilford Press; 2007.

4. Alarcon RD, Bell CC, Kirmayer J, Lin KM, Ustun B, Wisner KL. Beyond the funhouse mirror: research agenda on culture and psychiatric diagnosis. In: Kupfer DJ, First MB, Regier DA, eds. A Research Agenda for DSM-V. American Psychiatric Association;2002:219-281.

5. Alarcon RD, Becker AE, Lewis-Fernandez R, et al. Issues for DSM-V: the role of culture in psychiatric diagnosis. J Nerv Ment Dis. 2009;197(8):559-660. doi:10.1097/NMD.0b013e3181b0cbff

6. Lewis-Fernandez R, Aggarwal NK. Culture and psychiatric diagnosis. Adv Psychosom Med. 2013;33:15-30. doi:10.1159/000348725

7. Mezzich JE, Berganza CE, Ruiperez MA. Culture in Dsm-Iv, Icd10, and Evolving Diagnostic Systems. Psychiatr Clin North Am. 2001;24(3):407-419. doi:10.1016/s0193-953x(05)70237-9

8. Sharan $P$, Keeley J. Cultural perspectives related to international classification of Diseases-11. Indian J Soc Psychiatry. 2018;34(5):1. doi:10.4103/ijsp.ijsp_45_18

9. Reed GM, Sharan P, Saxena S. Reducing disease burden through the revision of ICD-10 mental and behavioural disorders. Natl Med J India. 2009;22(6):285-288.

10. Otero-Ojeda AA. Third Cuban Glossary of Psychiatry (GC-3): key features and contributions. Psychopathology. 2002;35(2-3):181184. doi:10.1159/000065142

11. Clark LA, Cuthbert B, Lewis-Fernandez R, Narrow WE, Reed GM. Three Approaches to Understanding and Classifying Mental Disorder: ICD-11, DSM-5, and the National Institute of Mental Health's Research Domain Criteria (RDoC). Psychol Sci Public Interest. 2017;18(2):72-145. doi:10.1177/1529100617727266

12. Reed GM, Mendonca Correia J, Esparza P, Saxena S, Maj M. The WPA-WHO Global Survey of Psychiatrists' Attitudes Towards Mental Disorders Classification. World Psychiatry. 2011;10(2):118131. doi:10.1002/j.2051-5545.2011.tb00034.x

13. Evans SC, Reed GM, Roberts MC, et al. Psychologists' perspectives on the diagnostic classification of mental disorders: results from the WHO-IUPsyS Global Survey. Int J Psychol. 2013;48(3):177-193. doi:10.1080/00207594.2013.804189

14. Reed GM, Rebello TJ, Pike KM, et al. WHO's Global Clinical Practice Network for mental health. Lancet Psychiatry. 2015;2(5):379-380. doi:10.1016/S2215-0366(15)00183-2

15. Keeley JW, Reed GM, Roberts MC, et al. Developing a science of clinical utility in diagnostic classification systems field study strategies for ICD-11 mental and behavioral disorders. Am Psychol. 2016;71(1):3-16. doi:10.1037/a0039972

16. First MB, Reed GM, Hyman SE, Saxena S. The development of the ICD-11 Clinical Descriptions and Diagnostic Guidelines for Mental and Behavioural Disorders. World Psychiatry. 2015;14(1):82-90. doi:10.1002/wps.20189

17. International Advisory Group for the Revision of ICDM, Behavioural D. A conceptual framework for the revision of the ICD-10 classification of mental and behavioural disorders. World Psychiatry. 2011;10(2):86-92. doi:10.1002/j.2051-5545.2011.tb00022.x 
18. Henrich J, Heine SJ, Norenzayan A. The weirdest people in the world?. Behav Brain Sci. 2010;33(2-3):61-135. doi:10.1017/ S0140525X0999152X

19. Gureje O, Stein DJ. Classification of mental disorders: the importance of inclusive decision-making. Int Rev Psychiatry. 2012;24(6):606-612. doi:10.3109/09540261.2012.726214

20. Gureje O, Lewis-Fernandez R, Hall BJ, Reed GM. Cultural considerations in the classification of mental disorders: why and how in ICD-11. BMC Med. 2020;18(1):25. doi:10.1186/s12916-020-1493-4

21. Raha B, Sarma S, Thilakan P, Punnoose ZM. Rumination Disorder: An Unexplained Case of Recurrent Vomiting. Indian J Psychol Med. 2017;39(3):361-363. doi:10.4103/0253-7176.207323

22. Gupta R, Kalla M, Gupta JB. Adult rumination syndrome: Differentiation from psychogenic intractable vomiting. Indian J Psychiatry. 2012;54(3):283-285. doi:10.4103/0019-5545.102434

23. Khandelwal SK, Sharan P, Saxena S. Eating disorders: an Indian perspective. Int J Soc Psychiatry. 1995;41(2):132-146. doi:10.1177/002076409504100206

24. International Advisory Group for the Revision of ICDM, Behavioural D. A conceptual framework for the revision of the ICD-10 classification of mental and behavioural disorders. World Psychiatry. 2011;10(2):86-92. doi:10.1002/j.2051-5545.2011.tb00022.x

25. American Psychiatric Association. Diagnostic and Statistical Manual of Mental Disorders. 5th ed. American Psychiatric Association; 2013.

26. Al-Adawi S, Bax B, Bryant-Waugh R, et al. Revision of ICD - status update on feeding and eating disorders. Adv Eat Disord Theory Res Pract. 2013;1(1):10-20. doi:10.1080/21662630.2013.742971

27. Gupta N, Malhotra N. Dissociative disorders: Reinvention or reconceptualization of the concept? Indian J Soc Psychiatry. 2018;34(5):44. doi:10.4103/ijsp.ijsp_40_18

28. Lewis-Fernandez R, Aggarwal NK. Culture and psychiatric diagnosis. Adv Psychosom Med. 2013;33:15-30. doi:10.1159/000348725

29. Lee S, Kleinman A. Are somatoform disorders changing with time? The case of neurasthenia in China. Psychosom Med. 2007;69(9):846849. doi:10.1097/PSY.0b013e31815b0092

30. Vaidyanathan S, Kuppili PP, Menon V. Eating Disorders: An Overview of Indian Research. Indian J Psychol Med. 2019;41(4):311-317. doi:10.4103/IJPSYM.IJPSYM_461_18
31. Mammen P, Russell S, Russell PS. Prevalence of eating disorders and psychiatric comorbidity among children and adolescents. Indian Pediatr. 2007;44(5):357-359.

32. Levy RL, Olden KW, Naliboff BD, et al. Psychosocial aspects of the functional gastrointestinal disorders. Gastroenterology. 2006;130(5):1447-1458. doi:10.1053/j.gastro.2005.11.057

33. Li BU, Balint JP. Cyclic vomiting syndrome: evolution in our understanding of a brain-gut disorder. Adv Pediatr. 2000;47:117-160.

34. Tack J, Talley NJ, Camilleri M, et al. Functional gastroduodenal disorders. Gastroenterology. 2006;130(5):1466-1479. doi:10.1053/j. gastro.2005.11.059

35. Talley NJ. Functional nausea and vomiting. Aust Fam Physician. 2007;36(9):694-697.

36. Groen SP, Richters A, Laban CJ, Deville WL. Implementation of the Cultural Formulation through a newly developed Brief Cultural Interview: Pilot data from the Netherlands. Transcult Psychiatry. 2017;54(1):3-22. doi:10.1177/1363461516678342

37. Groleau D, Young A, Kirmayer LJ. The McGill Illness Narrative Interview (MINI): an interview schedule to elicit meanings and modes of reasoning related to illness experience. Transcult Psychiatry. 2006;43(4):671-691. doi:10.1177/1363461506070796

38. Edwards LM, Burkard AW, Adams HA, Newcomb SA. A mixedmethod study of psychologists' use of multicultural assessment. Professional Psychology: Research and Practice. 2017;48(2):131-138. doi:10.1037/pro0000095

39. Kleinman AM. Depression, somatization and the "new crosscultural psychiatry". Soc Sci Med. 1977;11(1):3-10. doi:10.1016/00377856(77)90138-x

40. Prakash S, Sharan P, Sood M. A qualitative study on psychopathology of dhat syndrome in men: Implications for classification of disorders. Asian J Psychiatr. 2018;35:79-88. doi:10.1016/j.ajp.2018.05.007

41. Keshavan MS. Culture bound syndromes: disease entities or simply concepts of distress? Asian J Psychiatr. 2014;12:1-2. doi:10.1016/j. ajp.2014.11.003

42. Paralikar VP, Deshmukh A, Weiss MG. Qualitative Analysis of Cultural Formulation Interview: Findings and Implications for Revising the Outline for Cultural Formulation. Transcult Psychiatry. 2020;57(4):525-541. doi:10.1177/1363461518822407 